\title{
Tectonics in almost real time
}

\section{Very-Long-Baseline Interferometry has been used with remarkable precision to map the movement of the coastal strip of California. Has the time now come when other regions should follow suit?}

FANTASY has of course no place in science, but speculation may have a part to play. This is the justification for what follows, which is an account of how the movement of California west (or seawards) of the San Andreas Fault has been followed virtually instantaneously (over the few years since the technique became possible) with the help of mobile radiotelescopes operating in conjunction with a few fixed instruments in the same region. Those looking for a fuller account of what has been done should consult T.A.Clark et al., J. Geophys. Res. 92, 12,741;1987.

There is naturally nothing new about the notion that radio interferometry might have an important part to play in cataloguing the relative motions of the continents. At its simplest, the technique is simply that of old-fashioned rangefinding: put your eyes further apart by viewing a distant object through reflecting prisms at the end of a horizontal beam in front of you, and you will be able to measure distances more accurately than in unassisted binocular vision.

But if the object is infinitely distant, so that the greater separation of the binocular points of view does not assist the measurement of distance, you have a sporting chance of estimating the size of the distant object if you can collect phase as well as intensity information. And the better the precision, the more detailed the contour map of the emission intensity from the distant object that may be constructed. That, of course, is how maps of distant radiogalaxies are made.

So, turning the problem around, it is easy to see how the simultaneous observation of a distant fixed object by two radiotelescopes on the surface of the Earth can be used to estimate the distance between them. This has been an important objective of Very-Long-Baseline Interferometry (capitalized that way because the mnemonic is VLBI) for at least the past 15 years. There is a certain amount of breath being held by those who wish to know whether the Atlantic is realiy broadening by between 5 and $10 \mathrm{~cm}$ a year. The usual assumption has been that it would be necessary to use large and therefore fixed radiotelescopes to wring accurate geodetic information from radiointerferometric measurements, and that the best baselines would be very long, perhaps even interplanetary in scale. Clark et al. have a simpler tale to tell.

Everything hangs on the properties of what is called the Mark III geodetic VLBI system, devised by the US National Geodetic Survey in 1977. The system is not so much a technology as a method of management. Two independent radiotelescopes with receivers operating at two widely separate frequencies $(2.2 \mathrm{GHz}$ and $8.4 \mathrm{GHz}$ ) observe not just one distant radio source for just a few minutes, but several sources over several hours.

Naturally, the geodetic people cannot expect such dedication of time from the managers of radiotelescopes whose objectives are broader than the mere measurement of the Earth, which is why the National Geodetic Survey established a number of radiotelescopes in the United States with Mark III specifications that could be used for substantial periods of time on geodetic work. Clark et al. have the good luck to report on the usefulness of an extension of this network - a system using two mobile radiotelescopes which have been trucked from one side of the San Andreas Fault to the other during the past seven years.

The results of the survey of the movement of the western sliver of California are quite remarkable. For precision, the mobile telescopes have worked in conjunction with fixed telescopes scattered throughout the western United States. The mobile dishes are merely $3 \mathrm{~m}$ and $5 \mathrm{~m}$ in diameter, and can apparently be relocated to new sites at distances of several hundreds of kilometres at intervals of two days. Each convoy consists of two vans, one for the dish and one for the electronics. In the study of California west of the San Andreas Fault, the strategy has been to reoccupy the same observing site at regular intervals.

Unsurprisingly, it turns out that the coastal strip of California is indeed moving north-west relative to the continental United States at a lateral rate of $5.0 \mathrm{~cm}$ a year ( $\pm 0.1 \mathrm{~cm}$ a year). It is more remarkable that the inferred directions of movement of different parts of the western strip of California appear to be parallel to the most pronounced faults in the immediate neighbourhood, whether the San Andreas Fault or some other. No doubt it is only a matter of time before the operators of the mobile radiotelescopes are able to compete with the laser groundranging people in the accuracy with which they can tell which parts of the San Andreas Fault move smoothly, and which are locked together as a potential earth- quake source.It is particularly interesting that the inferred movements of the coastal strip relative to those of the fixed inland observatories are large enough to throw light on the changes taking place in the Basin and Range Province, the wasteland between the Rockies and the Sierras. Using a fixed point in the Mojave Desert (not nearly half-way between California and Arizona) as a reference point, Clark et al. conclude that, if coastal California is moving north-west at roughly $4 \mathrm{~cm}$ a year, Flagstaff (in Arizona) is moving in the opposite direction at roughly a third the speed. But if Arizona is the referencepoint, the total movement of the coastal strip is apparently sufficient to attribute all the relative motion of the Pacific and the North American tectonic plates to slip along the San Andreas Fault and its congeners, without the need to invoke slip along a parallel system of offshore faults.

The relatively small errors of these measurements are a consequence of two independent considerations - the amount of time for which the mobile stations have been dedicated to geodetic observation (and thus the amount of data collected per unit time) and the total length of time over which data have been accumulated (four years seems typical). There can hardly be a more striking illustration of how, in the exploitation of a new technique, management may be as important as technology.

Two questions immediately arise, of which the chief is whether the errors of these measurements with mobile dishes, small though they appear to be, can be further reduced. Obviously that must be possible. Provided that a mobile radiotelescope can see a specified distant radio source, what matters is chiefly that it should be able to record the time variation accurately. Sensitivity may be less important than the accuracy of timing.

So should not systems such as that described by Clark et al. find some kind of application in other tectonic areas? There are few places where the rate of local movement is as great as $5 \mathrm{~cm}$ a year, but it is not unreasonable to hope that movements of the order of $1 \mathrm{~cm}$ a year will soon be measurable over intervals of a few years. The great fault systems of Central and Eastern Asia should be readily measurable by these means. While disaster prevention is as much the rage as now, is that not something to plan for?

John Maddox 